\title{
A 27 - Gauge Microincision Vitrectomy Surgery for Posterior Segment Diseases in a Tertiary Centre, Nepal
}

\author{
Chunu Shrestha, Sabina Shrestha, Aparajita Manoranjan and Reeta Rajbhandari
}

Department of Ophthalmology, RM Kedia Eye Hospital, Birgunj, Nepal

\begin{abstract}
Introduction: Transconjunctival micro incision vitrectomy surgery (MIVS) with 23 or 25 gauge instrumentation is more advantageous than traditional 20 gauge surgery. We intended to evaluate the visual outcome, complications and indication of various vitreoretinal diseases using 27 Gauge vitrectomy systems.

Methods: This study was a prospective, interventional, non-comparative study conducted in Nepal Eye Hospital from June 2017 to July 2018. Fifty-two patients with various vitreoretinal diseases were recruited. The main outcome measures included corrected distance visual acuity (CDVA) preoperative and postoperative, intraocular pressure (IOP) preoperative and postoperative, indication for vitreoretinal surgery, intraoperative complications, and postoperative complications.

Results: Fifty two eyes of 52 patients (26 men and 26 women; mean age, 59 years) underwent 27 -gauge pars planavitrectomy. Surgical indications included epiretinal membrane $(n=11)$, full-thickness macular hole $(n=11)$, rhegmatogenous retinal detachment $[n=14$ (12 vitrectomy only and two vitrectomy with scleral buckle)], vitreous haemorrhage $(n=3)$, vitreous opacities $(n=3)$, silicon oil removal $(n=3)$, proliferative diabetic retinopathy $[\mathrm{n}=6$ (5 vitreous haemorrhage and one tractional retinal detachment)], posterior IOL dislocation $(\mathrm{n}=1)$. Postoperative complications included transient ocular hypertension in eight eyes $(15.39 \%)$, transient hypotony in five eyes $(9.62 \%)$ and vitreous haemorrhage in five eyes $(9.62 \%)$. No cases of postoperative endophthalmitis, sclerotomy - related tears, or choroidal detachments were encountered in the follow-up period. Mean corrected distance visual acuity improved from $20 / 796$ (logarithm of the minimum angle of resolution, $1.60 \pm 0.64$ ) preoperatively to $20 / 49$ (logarithm of the minimum angle of resolution, $0.42 \pm 0.26)$ postoperatively $(\mathrm{p}=0.000$, ) at final follow up.
\end{abstract}

Conclusions: 27-gauge micro-incision vitrectomy surgery was found to be a safe and effective sutureless surgery with favourable outcomes, in terms of vision.

Key words: 27 gauge vitrectomy; micro incision vitrectomy surgery (MIVS); small gauge vitrectomy; vitreoretinal surgery

Correspondence: Chunu Shrestha, RM Kedia Eye Hospital, Birgunj, Nepal. Email: chunu20@hotmail.com

DOI: $10.3126 / \mathrm{mjsbh.v20i2.30726}$

Submitted on: 2020-08-21

Accepted on: 2021-06-07

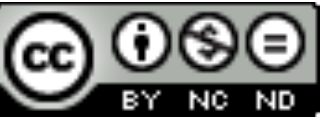

This work is licensed under creative common license:

http://creativecommons.org/licenses/by-nc-nd/4.0/ (C) MJSBH 2020 


\section{INTRODUCTION}

Transconjunctival micro incision vitrectomy surgery (MIVS) with 23 or 25 gauge instrumentation has provided potential advantages over traditional 20 gauge surgery. Advantages include faster - wound healing, less conjunctival scarring, decreased operating time, elimination of astigmatism, improved patient comfort, and less post-operative inflammation with early visual recovery. MIVS attributes to a self - sealing sclerotomy so there is no need for conjunctival peritomy and suturing.

In 2010, Oshima et al introduced a 27 - gauge vitrectomy with an incision size of $0.4 \mathrm{~mm} .{ }^{1}$ Scleral incision size for 27 - gauge vitrectomy systems is $0.4 \mathrm{~mm}$ thus; 27-gauge incisions are $20 \%$ smaller than 25 - gauge incisions. There is a greater likelihood that the wound will self-seal quickly, because of both smaller wound diameter and simpler, neater wound architecture, reducing the risk for vitreous prolapse, and permitting faster wound healing. Due to the smaller wound sizes, 27 - gauge vitrectomy may be associated with less inflammation and less postoperative pain.

Indications for 27 - gauge vitrectomy are expanding and there are no definite criteria regarding proper case selection. Each vitreoretinal surgeon should develop his or her preferences according to personal experience and expertise. More procedures can be performed safely and efficiently using 27 - gauge vitrectomy techniques. These latest advances in technology have transformed our capabilities and improved our methods for performing vitrectomies. To our knowledge, this is the first time to use 27 gauge pars plana vitrectomy in Nepal. So, this study was conducted to evaluate the visual outcome, complication, and indication of this procedure.

\section{METHODS}

This study was a prospective, interventional, noncomparative study conducted in Nepal Eye Hospital, Kathmandu from June 2017 to July 2018. The inclusion criteria for this study included eyes that underwent primary 27 - gauge PPV for various indications including epiretinal membrane, diabetic tractional retinal detachment, proliferative diabetic retinopathy, full-thickness macular hole, rhegmatogenous retinal detachment with or without proliferative vitreoretinopathy (PVR), vitreous hemorrhage, vitreous opacities, scleral fixation of the intraocular lens and complete postoperative follow-up of at least 12 weeks. Fifty two eyes of 52 patients met the inclusion criteria and underwent 27 - gauge vitrectomy for a variety of vitreoretinal disorders with a minimum of three months followup period. Exclusion criteria included a follow-up period of less than three months, patients judged to be incapable of postoperative posturing, a history of penetrating ocular trauma, proliferative vitreoretinopathy (PVR) of grade $\mathrm{C}$ or greater, and significant ocular comorbidities such as uveitis, uncontrolled glaucoma, and glaucoma filtration surgery. The main outcome measures included corrected distance visual acuity (CDVA) preoperative and postoperative, intraocular pressure (IOP) preoperative and postoperative, indication for vitreoretinal surgery, intraoperative complications, and postoperative complications. CDVA was recorded as a Snellen visual acuity and converted to the logarithm of the minimum angle of resolution $(\log M A R)$ equivalents for statistical analysis. Counting finger (CF) vision was defined as 2.1 $\log$ MAR and hand movement (HM) was defined as $2.4 \log$ MAR.

Technique: Surgery was performed under peribulbar or posterior sub-tenonanaesthesia by a single surgeon (CS). Surgical eyes were prepared using 5\% povidone - iodine. All surgeries were performed using the constellation vitrectomy $27+$ total plus Pak vitrectomy system (Alcon Laboratories). For this study, the machine was set with an initial aspiration of $0 \mathrm{~mm} \mathrm{Hg}$ moving linearly to $650 \mathrm{~mm} \mathrm{Hg}$ when the foot pedal is fully depressed, maintaining a fixed cut rate of 7500 cuts per minute (CPM). During surgery, IOP was controlled to $25 \mathrm{~mm} \mathrm{Hg}$. For posterior visualisation, EIBOS 2 (Haag-Streit Surgical) was used. Twenty seven - gauge cannulas were placed using a 1-stepnon-beveled technique with a trocarcannula system. The conjunctiva and tenon capsule was displaced anteriorly from the intended sclerotomy site with forceps. A trocar was inserted at an angle of approximately $90^{\circ}$ to the sclera, facing directly toward the vitreous cavity, or angled less than 90 degrees to the sclera parallel to the limbus. The angle was kept perpendicular to the 
surface as the cannula was inserted into the eye. The cannula was held in place with forceps and the trocar was removed. The first sclerotomy was made in the inferotemporal region, and a 27 - gauge infusion cannula was attached. Its position inside the vitreous cavity was verified using a 27 - gauge light pipe. After the infusion line was opened, cannulas were placed in the superotemporal and superonasal regions. Ocular examination was performed at the 1 - day; 1 - week, 1 - month and final follow up at three months. On each follow up ocular examination with Snellen visual acuity, applanation tonometry, and slit-lamp biomicroscopy was performed. Postoperative complications including hypotony, ocular hypertension, retinal detachment, endophthalmitis, and choroidal detachment were recorded. Hypotony was defined as a new onset IOP of six $\mathrm{mm} \mathrm{Hg}$ or less at any postoperative visit and ocular hypertension was defined as an IOP of $25 \mathrm{~mm} \mathrm{Hg}$ or more at any visit. After surgery, nine eyes $(17.31 \%)$ were left with a fluid-filled vitreous cavity, 24 eyes $(46.15 \%)$ with C3F8 gas, one eye (1.9\%) with air; and 18 eyes (34.62\%) were infused with 1000 centistoke silicon oil. Statistical analysis was done using SPSS 21 version. All parameters were statistically analysed using the paired t-test with a $95 \%$ confidence interval. A p - value of $<$ 0.05 considered to be statistically significant. The primary outcome measures were changed in visual acuity, indications and occurrence of intraoperative and postoperative complications.

\section{RESULTS}

In this study, 52 eyes met the inclusion criteria. Fifty two eyes of 52 patients $(26$ men and 26 women; mean age, 59 years) underwent 27 - gauge pars planavitrectomy. Surgical indications included epiretinal membrane $(\mathrm{n}=11)$, full-thickness macular hole $(\mathrm{n}=11)$, rhegmatogenous retinal detachment $[\mathrm{n}=14$ (12 vitrectomy only and two vitrectomy with scleral buckle)], vitreous haemorrhage $(\mathrm{n}=3)$, vitreous opacities $(\mathrm{n}=3)$, silicon oil removal $(\mathrm{n}=3)$, proliferative diabetic retinopathy $[n=6$ (Five vitreous haemorrhage and one tractional retinal detachment)], posterior IOL dislocation $(n=1)$.

Of the 52 eyes, 23 eyes underwent 27-G vitrectomy combined with phacoemulsification and aspiration

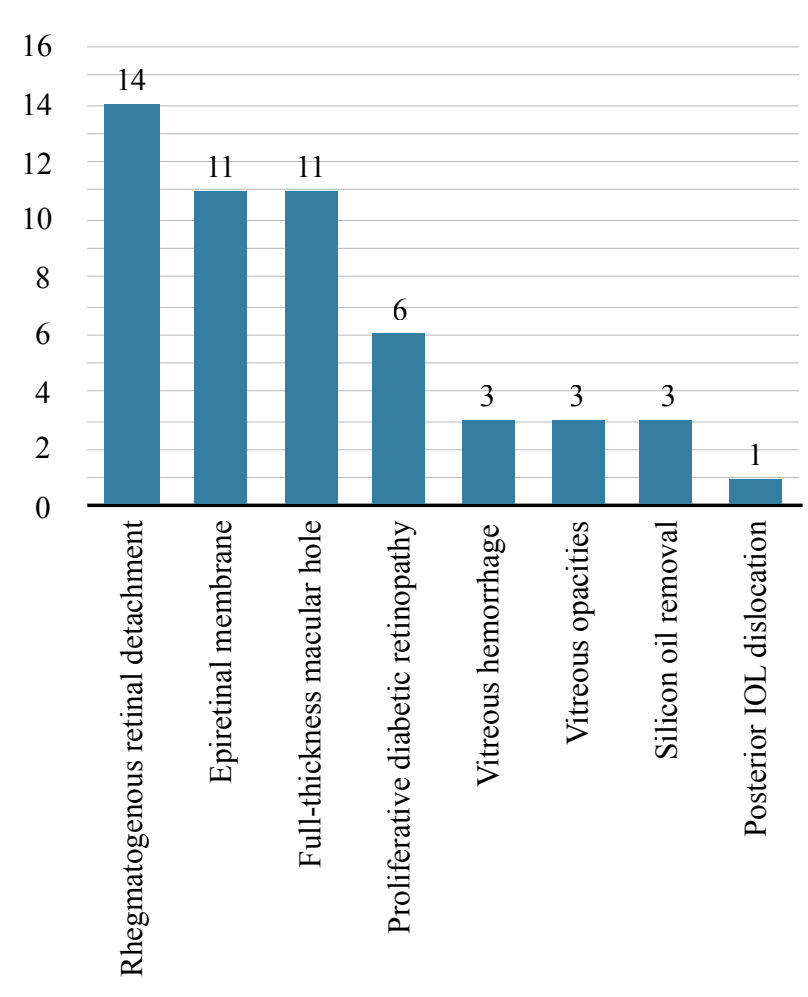

Figure 1. Surgical indication

and intraocular lens implantation, 14 pseudophakic eyes underwent $27-\mathrm{G}$ vitrectomy only, 14 phakic eyes underwent $27-\mathrm{G}$ vitrectomy only and one aphakic eye underwent $27-G$ vitrectomy and scleral fixation of intraocular lens implantation.

There were no intraoperative complications and no case required conversion to 20,23 , or 25 gauge instrumentation. Postoperative complications included transient ocular hypertension in eight eyes $(15.39 \%)$, transient hypotony in five eyes $(9.62 \%)$, and vitreous haemorrhage in five eyes $(9.62 \%)$. No cases of postoperative endophthalmitis, sclerotomy - related tears, or choroidal detachments were encountered in the follow-up period.

In all 14 eyes with rhegmatogenous retinal detachment, the retinal attachment was successfully

Table 1. Post-operative complication

\begin{tabular}{|l|r|r|}
\hline Complication & \multicolumn{1}{|c|}{$\begin{array}{c}\text { Frequency } \\
\text { (n) }\end{array}$} & $\begin{array}{c}\text { Percentage } \\
(\%)\end{array}$ \\
\hline $\begin{array}{l}\text { Transient ocular } \\
\text { hypertension }\end{array}$ & 8 & $15.39 \%$ \\
\hline Transient hypotony & 5 & $9.62 \%$ \\
\hline Vitreous hemorrhage & 5 & $9.62 \%$ \\
\hline
\end{tabular}


Table 2. Paired Sample Test of Difference in Preoperative and Final BCVA

\begin{tabular}{l|lll|l|l|l|l}
$\begin{array}{l}\text { Difference in } \\
\text { CDVA }\end{array}$ & Mean & Std. Deviation & $\begin{array}{l}\text { 95\% Confidence } \\
\text { interval (Lower) }\end{array}$ & $\begin{array}{l}95 \% \text { Confidence } \\
\text { interval (Upper) }\end{array}$ & $p$-value \\
\hline & 0.6089 & 1.1769 & 0.0844 & 13.937 & 0.000 \\
\end{tabular}

achieved by the initial surgery, and peripheral vitreous shaving was performed. Silicon oil (1000 cs) tamponade was performed in all 14 eyes $(100 \%)$. No postoperative complications were observed in all 14 eyes in this subgroup throughout the follow-up period. Twelve eyes underwent vitrectomy only and two eyes underwent vitrectomy with a scleral buckle. The mean preoperative visual acuity was 20 / 3899 (LogMAR, $2.29 \pm 0.14$ ), and it significantly improved to a mean CDVA of 20/56 (logMAR, $0.45 \pm 0.22)$ after the surgery $(\mathrm{P}=0.000)$. In all 11 eyes that underwent vitrectomy for an idiopathic ERM, the membrane was successfully removed without intra-operative complications, and no recurrence of membrane proliferation was observed during the study period. The mean preoperative visual acuity was $20 / 195(\log$ MAR, $0.99 \pm 0.14)$. The mean postoperative CDVA was $20 / 33$ $(\log$ MAR, $0.22 \pm 0.14)$, and the difference from preoperative value was statistically significant $(\mathrm{p}=$ $0.000)$

In all 11 eyes that underwent vitrectomy for an idiopathic $\mathrm{MH}$, successful closure of the hole was achieved via the initial surgery with the use of $16 \%$ perfluoropropane $(\mathrm{C} 3 \mathrm{~F} 8)$ as a tamponade. The mean visual acuity was 20/263 (logMAR, $1.12 \pm$ 0.28 ), yet it significantly improved to $20 / 50$ $(\log$ MAR, $0.40 \pm 0.33)$ at the final visit $(\mathrm{P}=0.000)$. Of the six eyes that underwent surgery for proliferative diabetic retinopathy (PDR), the reason for undergoing vitrectomy was non-resolving vitreous haemorrhage in five eyes and tractional retinal detachment in one eye. Silicon oil tamponade was performed in three eyes and gas tamponade was performed in three of the six eyes $(14 \%)$. The mean preoperative visual acuity was $20 / 29582(\log$ MAR, $3.17 \pm 0.40)$, yet it significantly improved to a mean postoperative CDVA of $20 / 632(\log M A R, 1.50 \pm 0.54)(\mathrm{p}=$ $0.001)$.

Of the three eyes that underwent surgery for vitreous haemorrhage, two eyes had branch retinal vein occlusion and one eye had trauma. The mean preoperative visual acuity was 20 / $6324(\log$ MAR, $2.50 \pm 0.17$ ), and the mean postoperative CDVA was $20 / 33(\log M A R, 0.23 \pm 0.05)$ with significant improvement $(\mathrm{p}=0.001)$. All the three eyes that underwent silicon oil removal was the case of retinal detachment done three months back. The

Table 3. Overall Patient Profile and Surgical Outcomes

\begin{tabular}{|c|c|c|c|c|}
\hline Diagnosis (n) & $\begin{array}{l}\text { Mean age, } \pm \\
\text { SD, Year }\end{array}$ & $\begin{array}{c}\text { Mean Pre, } \\
\text { LogMAR } \pm \text { SD }\end{array}$ & $\begin{array}{l}\text { Mean Post, } \\
\text { LogMAR } \pm \text { SD }\end{array}$ & p-value \\
\hline $\begin{array}{l}\text { Rhegmatogenous retinal detachment }(12+2=14) \\
(12 \text { Vitrectomy only }+2 \text { combined vitrectomy with } \\
\text { scleral buckle }\end{array}$ & $55.71 \pm 9.4$ & $2.29 \pm 0.14$ & $0.45 \pm 0.22$ & 0.000 \\
\hline Epiretinal membrane (11) & $65.91 \pm 4.7$ & $0.99 \pm 0.14$ & $0.22 \pm 0.14$ & 0.000 \\
\hline Full-thickness macular hole (11) & $64.45 \pm 5.9$ & $1.12 \pm 0.28$ & $0.40 \pm 0.33$ & 0.000 \\
\hline $\begin{array}{l}\text { Proliferative diabetic retinopathy }(5+1=6)(5 \\
\text { vitreous hemorrhage and } 1 \text { tractional retinal } \\
\text { detachment) }\end{array}$ & $51.60 \pm 9.0$ & $3.17 \pm 0.40$ & $1.50 \pm 0.54$ & 0.001 \\
\hline Vitreous hemorrhage (3) & $60.33 \pm 24.7$ & $2.50 \pm 0.17$ & $0.23 \pm 0.05$ & 0.001 \\
\hline Vitreous opacities (3) & $57.67 \pm 14.5$ & $1.43 \pm 0.90$ & $0.43 \pm 0.32$ & 0.211 \\
\hline Silicon oil removal (3) & $52.67 \pm 8.7$ & $2.00 \pm 0.34$ & $0.63 \pm 0.15$ & 0.028 \\
\hline Posterior IOL dislocation (1) & 63 & 2.10 & 0.20 & \\
\hline
\end{tabular}


mean preoperative visual acuity was 20/2000 (logMAR, $2.0 \pm 0.34$ ), and the mean postoperative CDVA was $20 / 85$ (logMAR, $0.63 \pm 0.15)$ with significant improvement $(\mathrm{p}=0.028)$.

\section{DISCUSSION}

Sutureless vitrectomy has become more and more popular with vitreoretinal surgeons in recent years. Oshima et al. introduced a 27 - gauge vitrector and demonstrated good surgical results. The 27 - gauge system allowed a safe approach to shaving the vitreous close to a mobile retina or when proximity to the retina was required to remove strong vitreoretinal adhesions. ${ }^{1}$ The advantages of sutureless surgery include minimisation of ocular trauma and suture-induced astigmatism, as well as the fact that postoperative inflammation is less. However, for complex cases, small-gauge suture-less vitrectomy systems may sometimes not be available or suitable. Disadvantages of small-gauge suture-less vitrectomy include prolong surgical time during vitrectomy, as well as increased flexibility of the smaller instruments. ${ }^{6-8}$

In cases undergoing suture-less vitrectomy, the reported range of postoperative hypotony is between $0 \%$ and $25 \% .{ }^{9,10}$ In our study, hypotony was observed in five eyes (9.62\%) which improved spontaneously within one week after the surgery. Yoneda et al ${ }^{11}$ observed hypotony in 15 eyes (9.2\%) which was similar to our study. M. Ali Khanet al. ${ }^{4}$ studied in ninety-five eyes and transient hypotony was seen in five eyes (5.3\%).

Transient ocular hypertension in eight eyes $(15.39 \%)$ was seen in our study and was treated successfully with oral anti-glaucoma medicine. Khan et $\mathrm{al}^{4}$ conducted a study that showed eight eyes (8.4\%) with transient ocular hypertension. Similar to Oshima et al. and Rizzo et al. ${ }^{1,5}$ No cases required conversion to larger-gauge instrumentation. There was no postoperative endophthalmitis, retinal tear secondary to sclerotomy, or choroidal detachment in our study. The choroidal detachment was observed in three eyes in a study done by Yoneda et al. ${ }^{11}$ Mean corrected distance visual acuity improved from 20/796 (logarithm of the minimum angle of resolution, $1.60 \pm 0.64$ ) preoperatively to $20 / 49$ (logarithm of the minimum angle of resolution, $0.42 \pm 0.26)$ postoperatively $(\mathrm{p}=0.000)$ at final follow up. Surgical indications were varied, including not only macular cases but also more complicated cases such as diabetic tractional retinal detachment and rhegmatogenous retinal detachment with PVR requiring silicon oil.

Although ours is a novel study from Nepal, we have to acknowledge some limitations. The major ones are that ours is a small sample sized study with short follow-up and we were not able to address the long-term complications related to the smaller-incision vitrectomy. However, we hope that our study would pave pathway for more elaborate studies in this field in the future.

\section{CONCLUSIONS}

27 - gauge micro-incision vitrectomy surgery is a safe and effective suture-less surgery as largergauge vitrectomy systems. 27-gauge micro-incision vitrectomy surgery has a favourable outcome, in terms of vision. The postoperative complications are very few which can be managed easily. It offers earlier postoperative visual recovery.

To cite this article: Shrestha C, Shrestha S, Manoranjan A, Rajbhandari R. A 27 - Gauge Microincision Vitrectomy Surgery for Posterior Segment Diseases in a Tertiary Centre, Nepal. 2021;20(2):91-6.

Conflict of Interest: None declared

\section{REFERENCES}

1. Oshima Y, Wakabayashi T, Sato T, Ohji M, Tano Y. A 27-gauge instrument system for transconjunctival sutureless microincision vitrectomy surgery. Ophthalmology. 2010 Jan;117(1):93-102. DOI:10.1016/j.ophtha.2009.06.043. 
2. Mitsui K, Kogo J, Takeda H, Shiono A, Sasaki H, Munemasa Y, et al. Comparative study of 27-gauge vs 25-gauge vitrectomy for epiretinal membrane. Eye (Lond). 2016 Apr;30(4):538-44. DOI: 10.1038/eye.2015.275.

3. Nagiel A, McCannel CA, Moreno C, McCannel TA. Vitrectomy assisted biopsy for molecular prognostication of choroidal melanoma $2 \mathrm{~mm}$ or less in thickness with a 27-gauge cutter. Retina. $2017 \mathrm{Jul} ; 37(7): 1377-82$. DOI: 10.1097/IAE.0000000000001362.

4. Khan MA, Shahlaee A, Toussaint B, Hsu J, Sivalingam A, Dugel PU, et al. Outcomes of 27 Gauge Microincision Vitrectomy Surgery for Posterior Segment Disease. Am J Ophthalmol. 2016 Jan;161:36-43.e1-2. DOI: 10.1016/ j.ajo.2015.09.024.

5. Rizzo S, Barca F, Caporossi T, Mariotti C. Twenty-seven-gauge vitrectomy for various vitreoretinal diseases. Retina. 2015 Jun;35(6):1273-8. DOI: 10.1097/IAE.0000000000000545.

6. Lee JE, Kim KH, Kim IK, Jea SY, Kim WS. Comparison of 20-gauge transconjunctival sutureless vitrectomy with conventional vitrectomy. Retina. 2010 Oct;30(9):1496-504. DOI: 10.1097/IAE.0b013e3181d427d3.

7. Gotzaridis EV. Sutureless Transconjunctival 20 Gauge pars plana Vitrectomy. Semin Ophthalmol. 2007;22(3): 179-83. DOI: 10.1080/08820530701501170.

8. Kim JE, Shah SN, Choi DL, Han DP, Connor TB. Transconjunctival 20-gauge pars plana vitrectomy using a single entry cannulated sutureless system. Retina. 2009 Oct;29(9):1294-8. DOI: 10.1097/IAE.0b013e3181aa8e3b.

9. Teixeira A, Allemann N, Yamada AC, Uno F, Maia A, Bonomo PP. Ultrasound biomicroscopy in recently postoperative 23-gauge transconjunctival vitrectomy sutureless self-sealing sclerotomy. Retina. 2009 Oct;29(9): 1305-9. DOI: 10.1097/IAE.0b013e3181b09487.

10. Scott IU, Flynn HW, Dev S, Shaikh S, Mittra RA, Arevalo JF, et al. Endophthalmitis after 25-gauge and 20-gauge pars plana vitrectomy: incidence and outcomes. Retina. 2008 Jan;28(1):138-42. DOI: 10.1097/IAE. 0b013e31815e9313.

11. Yoneda K, Morikawa K, Oshima Y, Kinoshita S, Sotozono C. Japan Microincision Vitrectomy Surgery Study Group. Surgical outcomes of 27- G vitrectomy for a consecutive series of 163 eyes with various vitreous diseases. Retina. 2017 Nov;37(11):2130-7. DOI: 10.1097/IAE.0000000000001442. 